\title{
Characterization of in-depth cavity distribution after thermal annealing of helium-implanted silicon and gallium nitride
}

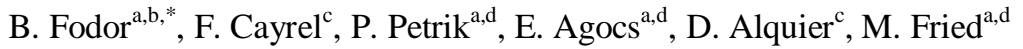 \\ ${ }^{a}$ Institute for Technical Physics and Materials Science, Research Centre for Natural Sciences (MTA TTK MFA), H-1121 Budapest, \\ Konkoly Thege u. 29-33, Hungary \\ ${ }^{b}$ Faculty of Science, University of Pécs, 7624 Pécs, Ifjúság útja 6, Hungary \\ ${ }^{c}$ GREMAN, pôle MTECH,Université François Rabelais, 16, rue Pierre et Marie Curie, B.P. 7155, F37071 Tours Cedex, France \\ ${ }^{d}$ Doctoral School of Molecular- and Nanotechnologies, Faculty of Information Technology, University of Pannonia, Egyetem u. 10, \\ Veszprem, H-8200, Hungary
}

*Corresponding author. Electronic address: fodor.balint@ @ttk.mta.hu

$+36205202592$

\section{Abstract}

Single-crystalline silicon wafers covered with sacrificial oxide layer and epitaxially grown gallium nitride layers were implanted with high-fluence helium ions $\left(2-6 \times 10^{16} \mathrm{~cm}^{-2}\right)$ at energies of $20-30 \mathrm{keV}$. Thermal annealings at $650-1000{ }^{\circ} \mathrm{C}, 1$ hour were performed on the Si samples and rapid thermal annealings at $900-1150{ }^{\circ} \mathrm{C}, 60-180 \mathrm{sec}$ under $\mathrm{N}_{2}$ were performed on the GaN samples. The as-implanted samples and the near-surface cavity distributions of the annealed samples were investigated with variable angle spectroscopic ellipsometry. In-depth defect profiles and cavity profiles can be best described with multiple independent effective medium sublayers of varying ratio of single-crystal/void. The number of sublayers was chosen to maximize the fit quality without a high parameter cross-correlation. The dependence of the implantation fluence, oxide layer thickness and annealing temperature on the cavity distribution was separately investigated. The ellipsometric fitted distributions were compared and cross-checked with analyses of transmission electron micrographs where the average surface cavity was determined sublayer by sublayer. The in-depth profiles were also compared with simulations of He and vacancy distributions.

Keywords:

Spectroscopic Ellipsometry; ion-implantation; annealing; cavity; depth profile

\section{Introduction}

High-dose helium implantation followed by thermal annealing leads to extended defects formations, such as dislocations and cavities in single-crystalline silicon (c-Si) and gallium nitride $(\mathrm{GaN})$. Cavities in these materials can be used for different applications, such as gettering of impurities during device processing, smart cut process or the diffusion control of dopants for ultrashalow junctions [1, 2, 3, 4, 5]. A further interest of He implantation induced defects in GaN concerns the formation of resistive guard rings in a schottky diode process [6] Transmission Electron Microscopy (TEM) is an established tool to investigate these structures, but unfortunately it is very time consuming and difficult to determine at a same time a depth distribution profile of the cavities and an observation when defects of few $\mathrm{nm}$ to thousands nm are encountered in the same area. These structures can be studied by Spectroscopic Ellipsometry (SE) and, with appropriate multilayered models, the in-depth profiles of the implantation caused amorphization of $\mathrm{Si}$ and the cavity formation after thermal annealing can be evaluated $[7,8,9,10]$. SE has the advantage over TEM that it is fast, non destructive and so can be used as a feedback control during industrial process. In this study a large number of $\mathrm{Si}$ wafers and $\mathrm{GaN}$ layers subjected to different implantation and annealing conditions are investigated. The cavities formation in $\mathrm{Si}$ is investigated as a function of the implantation dose, the annealing temperature and the thickness of a sacrificial oxide layer. Furthermore the well established multilayered model is utilized to evaluate the damaged $\mathrm{GaN}$ as well.

\section{Experimental details}

The sample preparations, the implantations and the annealings, as well as the TEM observations were made at GREMAN institute. The ellipsometric measurements and evaluations were made at MTA TTK MFA. Three sets of samples were prepared and analysed: Silicon wafers, $\mathrm{GaN}$ on Silicon and GaN on Sapphire substrates.

Single crystalline silicon $p$-type Czochralski (111) substrates, covered with a sacrificial oxide layer (1300, 1500 and $1700 \AA$ ), were implanted at $7^{\circ}$ tilt with high helium fluences $\left(2-6 \times 10^{16} \mathrm{~cm}^{-2}\right)$ at an energy of $20 \mathrm{keV}$. The $\mathrm{SiO}_{2}$ layers were removed by chemical etching in a $10 \%$ hydrofluoric acid solution. The samples were thermally annealed (conventional FA) under $\mathrm{N}_{2}$ atmosphere at 650,800 and $1000{ }^{\circ} \mathrm{C}$ for 1 hour. These samples will be referred as $\mathrm{SiO}_{2} / \mathrm{Si}$ samples.

$\mathrm{GaN}$ on Sapphire samples consist in a stack of " $\mathrm{n}$ - GaN $(9 \mu \mathrm{m}) /$ $\mathrm{n}^{+} \mathrm{GaN}(3 \mu \mathrm{m}) / \mathrm{AlGaN}-\mathrm{GaN}$ buffer $(3.2 \mu \mathrm{m})$ " epitaxially grown (MOCVD) on sapphire. These samples were implanted with $30 \mathrm{keV} \mathrm{He}$ ions at a fluence of $6 \times 10^{16} \mathrm{~cm}^{-2}$, afterwards they were subjected to [600 - 1000] ${ }^{\circ} \mathrm{C}$ rapid thermal annealing (RTA) for 2 minutes while covered with a $200 \mathrm{~nm}$ TEOS cap-layer. These samples will be referred as GaN/Sapphire samples.

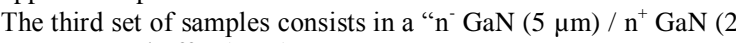
$\mu \mathrm{m}) / \mathrm{AlGaN}-\mathrm{GaN}$ buffer $(5 \mu \mathrm{m})$ " stack epitaxially grown (MOCVD) on Silicon. These samples were implanted with three different $\mathrm{He}$ ion fluences at $30 \mathrm{keV}$. Different annealing temperatures and durations have been chosen for these wafers. Following the previous conventions, these samples will be called in the article as GaN/Si samples. Table 1 summarizes the implantation and annealing conditions for all the investigated samples.

Table 1: Implantation and annealing conditions for the three investigated sample types

\begin{tabular}{|c|c|c|c|}
\hline Sample type & $\mathrm{SiO}_{2} / \mathrm{Si}$ & GaN/Sapphire & $\mathrm{GaN} / \mathrm{Si}$ \\
\hline $\begin{array}{c}\mathrm{SiO}_{2} \\
\text { or } \\
\mathrm{n}-/ \mathrm{n}+\mathrm{GaN} / \text { buffer } \\
\text { thickness }\end{array}$ & $\begin{array}{l}130 \mathrm{~nm} \\
150 \mathrm{~nm} \\
170 \mathrm{~nm}\end{array}$ & $9 / 3 / 3.2 \mu \mathrm{m}$ & $5 / 2 / 5 \mu \mathrm{m}$ \\
\hline Implantation energy & $20 \mathrm{keV}$ & $30 \mathrm{keV}$ & $30 \mathrm{keV}$ \\
\hline $\begin{array}{l}\text { Implantation } \\
\text { fluence }\end{array}$ & $\begin{array}{l}2 \times 10^{16} \mathrm{~cm}^{-2} \\
4 \times 10^{16} \mathrm{~cm}^{-2} \\
6 \times 10^{16} \mathrm{~cm}^{-2}\end{array}$ & $6 \times 10^{16} \mathrm{~cm}^{-2}$ & $\begin{array}{l}\text { non-implanted } \\
1 \times 10^{16} \mathrm{~cm}^{-2} \\
6 \times 10^{16} \mathrm{~cm}^{-2}\end{array}$ \\
\hline $\begin{array}{l}\text { Annealing } \\
\text { temperature }\end{array}$ & $\begin{array}{l}\text { as-implanted } \\
6500^{\circ} \mathrm{C} \\
800{ }^{\circ} \mathrm{C} \\
1000{ }^{\circ} \mathrm{C}\end{array}$ & $\begin{array}{l}600^{\circ} \mathrm{C} \\
700{ }^{\circ} \mathrm{C} \\
800{ }^{\circ} \mathrm{C} \\
900{ }^{\circ} \mathrm{C} \\
1000^{\circ} \mathrm{C}\end{array}$ & $\begin{array}{l}\text { as-implanted } \\
9500^{\circ} \mathrm{C} \\
1000{ }^{\circ} \mathrm{C} \\
1100{ }^{\circ} \mathrm{C}\end{array}$ \\
\hline Annealing time & $60 \mathrm{~min} F A$ & $2 \min \mathrm{RTA}$ & $\begin{array}{l}1 \min \text { RTA } \\
3 \min \text { RTA }\end{array}$ \\
\hline Number of samples & 37 & 7 & 15 \\
\hline
\end{tabular}

The SE measurements were performed on the non-implanted, the as-implanted and the annealed samples using a Woollam M-2000DI variable angle spectroscopic ellipsometer. It is a rotating compensator ellipsometer with a multichannel detection system. This setup enables the measurement of the ellipsometric angles with an accuracy of $5 \times 10^{-2}$ for both $\Psi$ and $\Delta$. The measurements were performed at an angle of incidence between $70^{\circ}$ and $78^{\circ}$ for the $\mathrm{SiO}_{2} / \mathrm{Si}$ type samples and between $66^{\circ}$ and $70^{\circ}$ for the GaN thin layer type samples, in the wavelength range from 193 to $1690 \mathrm{~nm}$, with a spectral resolution of 1 $\mathrm{nm}$. These incident angles were chosen in order to correspond to the Brewster angle for the $\mathrm{Si}$ or the $\mathrm{GaN}$ for some wavelength within the spectral range. The recorded ellipsometric spectra were evaluated with CompleteEASE v4.72 and with WVASE v3.386 data acquisition and analysis software. These software use regression analysis to fit the free parameters of our optical models by the minimization of a well defined merit of fit, in our case the $\chi^{2}$, defined the following way:

$$
\chi^{2}=\frac{1}{N-P-1} \sum_{j=1}^{N}\left\{\left(\frac{\Delta_{j}^{\text {meas }}-\Delta_{j}^{\text {calc }}}{\sigma_{\Psi_{j}}^{\text {meas }}}\right)^{2}+\left(\frac{\Psi_{j}^{\text {meas }}-\Psi_{j}^{\text {calc }}}{\sigma_{\Delta j}^{\text {meas }}}\right)^{2}\right\},
$$


where $\Psi$ and $\Delta$ are the measured ('meas') and calculated ('calc') ellipsometric angles, $N$ is the number of independently measured values, $P$ is the number of unknown model parameters; $\sigma$ is the measurement error serving for the weighting of the difference of the measured and calculated values in the numerators.

Crystalline structures of the implanted samples were characterized using (scanning) transmission electron microscopy (STEM OR TEM), to investigate the material amorphisation or the distribution of cavities within the different materials $(\mathrm{Si} / \mathrm{GaN})$ after annealing. TEM lamella were prepared and observed with an "FEI Strata 400" dual-beam system (secondary electron microscopy (SEM) and focused ion beam (FIB)) equipped with a flip stage for lamella transfer on TEM grid and with a STEM detector for observations. A JEOL $2100 \mathrm{~F}$ was used in classical observation modes for TEM observations.

\section{Modelling and evaluations}

The ellipsometric models describing the 3 different kinds of annealed samples as well as the as-implanted counterpart followed a similar pattern. A surface native oxide layer, an amorphous or cavity layer and a semi infinite substrate layer was used for the $\mathrm{SiO}_{2} / \mathrm{Si}$ samples, while a simpler model, a defected layer and a semi infinite substrate layer was used for the GaN/Sapphire and GaN/Si samples. To account for the optical response of the intermediate layer (partially amorphous, or defected layer) several independent sublayers with varying fraction of component content was used with the help of the Bruggeman Effective Medium Approximation (EMA): A mixture of c$\mathrm{Si}$ and $\mathrm{a}-\mathrm{Si}$ for the amorphous region of the as-implanted $\mathrm{Si}$ samples (see inset in Figure 1.b) and a mixture of $\mathrm{c}-\mathrm{Si}$ and void for the cavity region of the annealed Si samples, as it was previously demonstrated to be a very good model choice [7, 8]. The dielectric function of the c-Si and $\mathrm{SiO}_{2}$ were taken from reference measurements from the literature. The dielectric function of the a-Si component was described with a single Tauc-Lorentz oscillator model using 4 independent fit parameters. As for the GaN/Sapphire and GaN/Si samples (see inset in Figure 8), the defected regions of the implanted and annealed $\mathrm{GaN}$ thin layers were considered to be a composition of reference $\mathrm{GaN}$ measured before implantation (non-implanted $\mathrm{GaN}$ ), of reference implanted $\mathrm{GaN}$ measured after implantation but before annealing (as-implanted $\mathrm{GaN}$ ) and of void. These multilayered models can describe a vertically inhomogeneous dielectric function and, due to the EMA volume fraction fit parameters, the in-depth distribution of the substituent content can be obtained (defect or void distribution). The thickness values of these sublayers were coupled to each other, while only the thickness of the whole amorphous or cavity layer was fitted. The substituent volume fractions were fitted independently. So for example a cavity layer of 5 sublayers has 5 void volume fractions and 1 thickness parameter, and of course the thickness parameter of the native oxide layer, totalling in 7 independent fit parameters.

The number of EMA sublayers $(N)$ has been chosen in a way to maximize fit quality while avoiding large fit errors $(>5 \%)$ and unphysical fit parameters. A large cross correlation (CC) between two parameters $(>98 \%)$ is also an indication, that our model is over parameterized, and so we cannot give credit to these fitted values as being representative of the sample. An example of how $N$ effects fit quality, maximum relative errors and the cross correlations for the evaluation of $\mathrm{SiO}_{2} / \mathrm{Si}$ type sample of $130 \mathrm{~nm}$ sacrificial oxide, implanted with $4 \times 10^{16} \mathrm{~cm}^{-2}$ and annealed at $800{ }^{\circ} \mathrm{C}$ are represented in Table 2. As it can be seen, when increasing the number of sublayers, the $\chi^{2}$ decreases. At $N=2-6, \chi^{2}$ decreases drastically, then it is less impacted by $N$. At $N=8$ large $\mathrm{CC}$ appears but the largest error is still acceptable, at $N=10, \mathrm{CC}$ and errors are too large.

Table 2: $\chi^{2}$, largest relative fit errors and largest absolute value of $\mathrm{CC}$ as a function of $\mathrm{N}$

\begin{tabular}{c|l|l|l|l|l|l|l|l|l}
$\mathbf{N}$ & 2 & 3 & 4 & 5 & 6 & 7 & 8 & 9 & 10 \\
\hline$\chi^{2}$ & 15.38 & 10.5 & 5.44 & 2.763 & 1.981 & 1.895 & 1.802 & 1.794 & 1.737 \\
$\begin{array}{c}\text { max. rel. } \\
\text { err. } \\
\text { max. } \\
\text { |CC| }\end{array}$ & $54 \%$ & $48 \%$ & $44 \%$ & $56 \%$ & $73.7 \%$ & $89.5 \%$ & $99.2 \%$ & $99.9 \%$ & $99.5 \%$
\end{tabular}

The cavities from the TEM micrographs were analysed the following way: each cavity layer was sliced into several sublayers ( 3 or 6). In each sublayer the surface projection of the cavities were added up, then divided by the total surface of the sublayer. This way a depth distribution of the cavities has been obtained (depth distribution of 2D cavity projection). Several cavities were cut into half on the image by two bordering sublayers. In this case, only the fraction of its surface belonging to the sublayer in question was counted into the sum of the surface of the cavities. For each sample, this has been done on three different micrographs to obtain average results. It is important to note, that the conversion from cavity profiles of $2 \mathrm{D}$ projected area to $3 \mathrm{D}$ volumetric cavity profiles is not straightforward. In the simple case when the distribution of cavities is homogeneous in depth and the cavities are sphere like, then with the following simple formula one can calculate the volume ratio: $V=\frac{A}{\left(\frac{\pi}{\Delta R}+1\right]}$, here $A$ stands for the area ratio, $d$

is the cross sectional thickness of the sample used in the analyses and $R$ is the average radius of the spheres. The first problem is that it is very difficult to know exactly the value of $d$. Also, the cross section of the sample can be wedge-shaped, meaning that $d$ can be different from sublayer to sublayer, further complicating the conversion. And lastly, the cavities are clearly not distributed homogenously as we will see from the evaluations. Because of these complications, cavity profiles are only compared between the original projected area of TEM analysis's and the volumetric SE evaluations. These remarks evidence the interest of Spectroscopic ellipsometry for such a type of analysis.

\section{Discussion and results}

\subsection{Evaluation of as-implanted samples}

Simulations with "The Stopping and Range of Ions in Matter" (SRIM) [11] of He and vacancy distributions in Si due to implantation through $\mathrm{SiO}_{2}$ are shown in Figure 1.a, beneath it the evaluation of the multilayered EMA model of the corresponding SE measurement is shown. It can be seen that the damage profile is very similar to the vacancy distribution but the damage profile has its peak closer to the surface, with a $94 \%$ amorphous content which is decreasing in depth. At the surface interface, there is a clear $7 \mathrm{~nm}$ thick layer that is totally amorphous, also reported by previous study [12]

a)

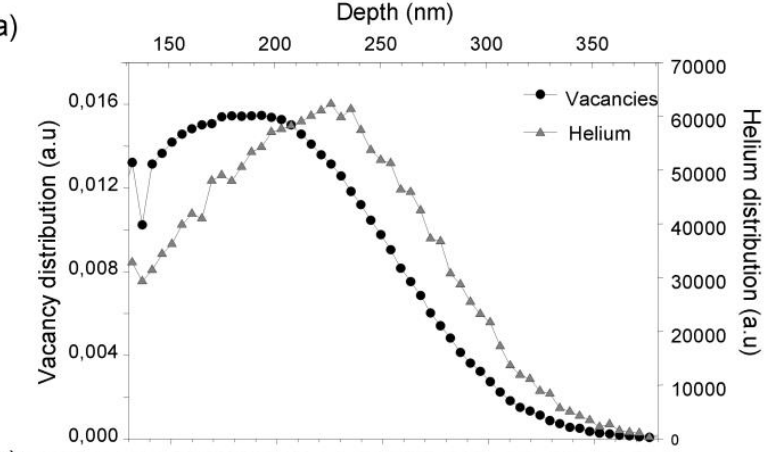

b)

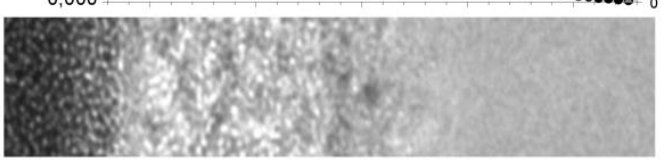

c)

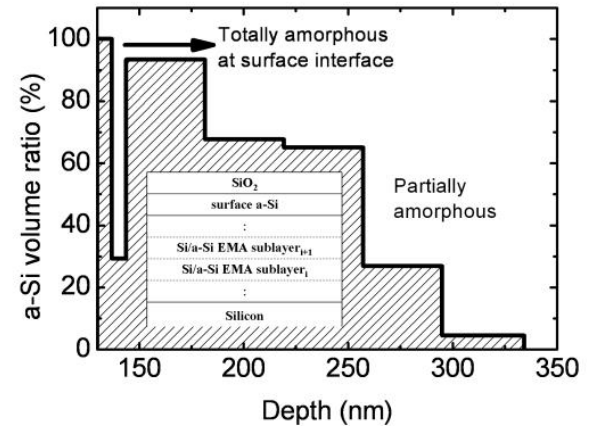

Figure 1: a) He and vacancy depth distribution from simulations for $20 \mathrm{keV}$, $4 \times 10^{16} \mathrm{~cm}^{-2}$ implantation through $130 \mathrm{~nm}$ sacrificial oxide layer, b) cross-sectional TEM image and c) amorphous volume fraction depth distribution from SE evaluation and ellipsometric model inset.

\subsection{Description of cavity formation in $\mathrm{Si}$ and its implantation and annealing dependencies}

As we increase the number of sublayers in the ellipsometric models, we obtain better depth resolution. For the TEM analysis maximum sublayer number depends on the size of the cavities (max 6), while for the SE evaluations it depends on the errors of the fitted values. In Figure 2 we can see the effect of increasing $N$. Left series correspond to the $800{ }^{\circ} \mathrm{C}$ annealing, right series to the $1000{ }^{\circ} \mathrm{C}$ annealing of the $\mathrm{SiO}_{2} / \mathrm{Si}$ samples with $130 \mathrm{~nm}$ sacrificial oxide implanted at $4 \times 10^{16} \mathrm{~cm}^{-2}$ dose. The top two images are small portions of TEM images displaying cavity morphology, beneath them the corresponding cavity surface depth distribution analysed on larger scales are shown. The bottom three graphs are the void depth distribution of $\mathrm{SE}$ evaluation fitted with $\mathrm{N}=3$, 6 , and 9, showing an increasing depth resolution. Clearly, SE depth 
profiles are very similar to those made by TEM analyses, which confirms the validity of the SE evaluations for cavity depth profiles in our case. Furthermore the cavity layer thicknesses are in very good agreement between the two methods (within $10 \mathrm{~nm}$ ), but the SE evaluations show a slightly larger thickness as it is more sensitive to smaller void fractions at the bottom interface, when using larger $N$. Although our SE model cannot describe cavity size or size distribution, it can have a better depth resolution than those obtained from TEM images. For some of the samples, where cavities are too small or not well separated as seen on the TEM micrographs, 2D cavity content is impossible to obtain. In these cases SE evaluations can still account for the volume content of these cavities from the fitting of multilayered EMAs.
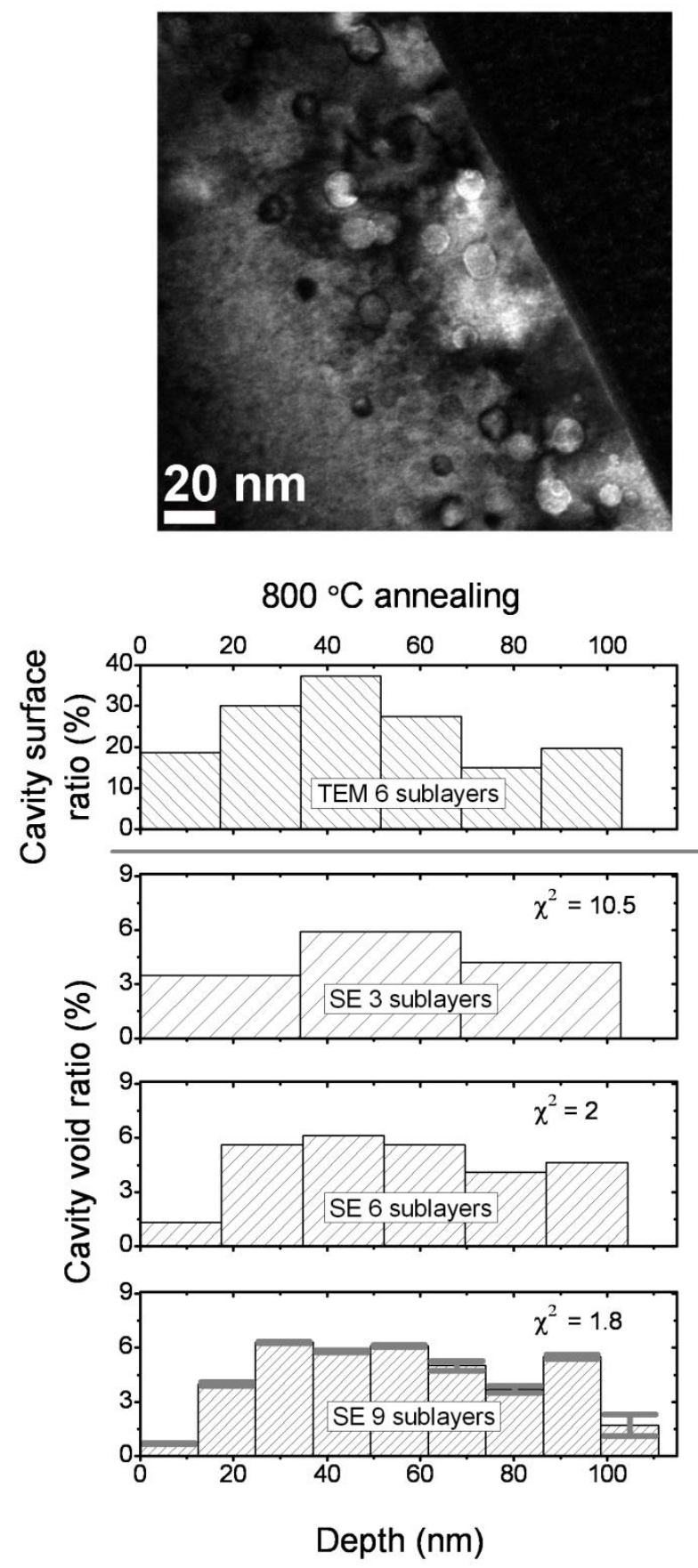

$130 \rightarrow 150 \rightarrow 170 \mathrm{~nm}$ oxide layer increase, a shift of $114 \rightarrow 87 \rightarrow 55$ $\mathrm{nm}$ for the depth of the cavity layer is observed, respectively. While the maximum remains relatively the same at $\sim 6 \%$ void, there is a slight sharpening at the bottom interface. The total cavity volume changes the following way: $480 \rightarrow 373 \rightarrow 253 \mathrm{~nm} \times$ unit surface. This shift is observable for all the other implantation and annealing conditions, but for the low dose implantation $\left(2 \times 10^{16} \mathrm{~cm}^{-2}\right)$ through the $170 \mathrm{~nm}$ oxide, the cavity layer from the SE evaluations reveal no depth structure and only a very small $(\sim 1 \%)$ void near the surface region. This can be clearly explained by both the low density of initial vacancy-helium clusters due to the low He fluence and by the surface proximity allowing higher exodiffusion of $\mathrm{He}$ and surface absorption of the vacancies.

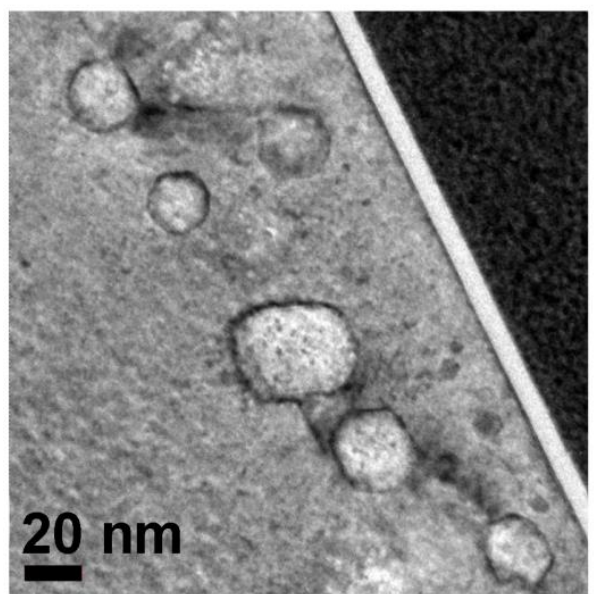

\section{$1000^{\circ} \mathrm{C}$ annealing}
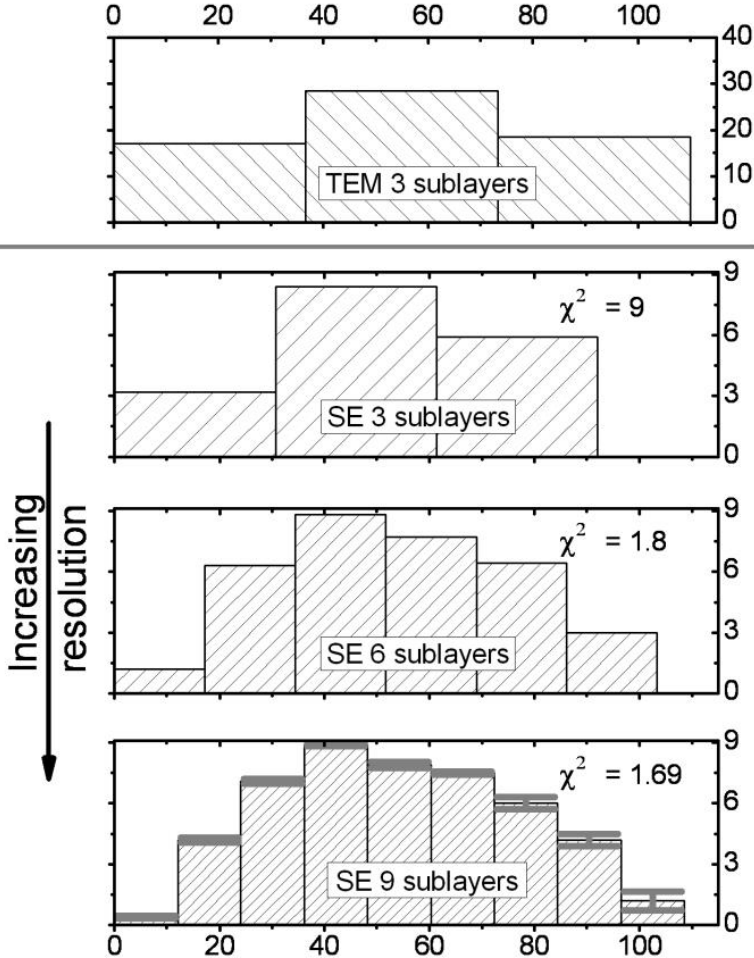

Depth (nm)

Figure 2: Cavity surface depth distribution of TEM analyses for the top two graphs, beneath them the void depth distribution of SE evaluation fitted with 3,6 , and 9 number of sublayers showing an increasing depth resolution. Left series corresponds to the $800{ }^{\circ} \mathrm{C}$ annealing, right series to the $1000{ }^{\circ} \mathrm{C}$ annealing of the $\mathrm{SiO} / \mathrm{Si}_{2}$ samples with $130 \mathrm{~nm}$ sacrificial oxide implanted at $4 \times 10^{16} \mathrm{~cm}^{-2}$ dose

One of the most apparent effects on cavity formation is the thickness of the sacrificial oxide layer through which the He radiations have been made. There is obviously a decrease of the cavity layer depth and also of the total cavity layer thickness when increasing the oxide thickness as demonstrated in Figure 3 for the $\mathrm{SiO}_{2} / \mathrm{Si}$ samples implanted with $4 \times 10^{16} \mathrm{~cm}^{-2}$ fluence and annealed at $800^{\circ} \mathrm{C}$. In this case for the 


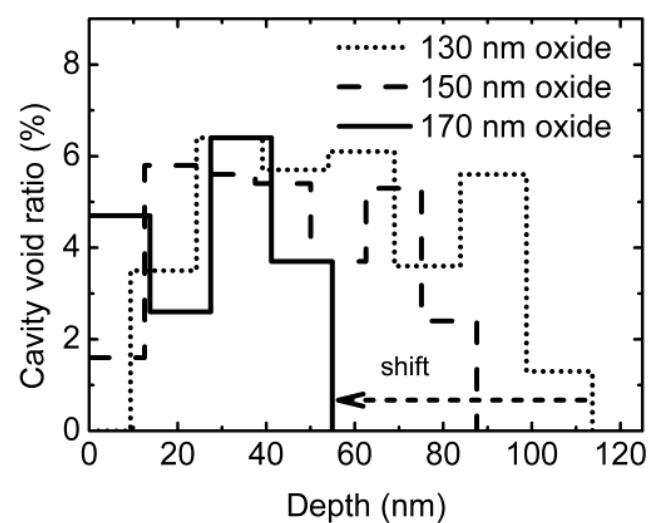

Figure 3: Sacrificial oxide dependence of the cavity distribution of the $4 \times 10^{16} \mathrm{~cm}$ implanted $800{ }^{\circ} \mathrm{C}$ annealed $\mathrm{SiO}_{2} / \mathrm{Si}$ samples.

The second effect of our study on the cavity layer structure is the implantation dose. In Figure 4 we can see the change of cavity void distribution for an increase of the implantation fluence demonstrated on the sample covered with $130 \mathrm{~nm}$ sacrificial oxide layer and annealed at $800^{\circ} \mathrm{C}$. There is a very high increase in the void density when the ion fluence changes from 2 to $6 \times 10^{16} \mathrm{~cm}^{-2}$ (low, medium and high dose). But more surprisingly, a shift of the peak from the bottom interface to the upper part of the cavity layer can also be seen. The total cavity volume changes the following way: $164 \rightarrow 480 \rightarrow 1398 \mathrm{~nm} \times$ unit surface. These changes in the cavity distribution as a function of the ion fluence can be seen for the other samples as well. A further general tendency is that the peaks are more broadened and less distinctive for the medium dose and these peaks tend to reach entirely the surface interface at high dose for the samples covered with 150 or $170 \mathrm{~nm}$ sacrificial oxide.

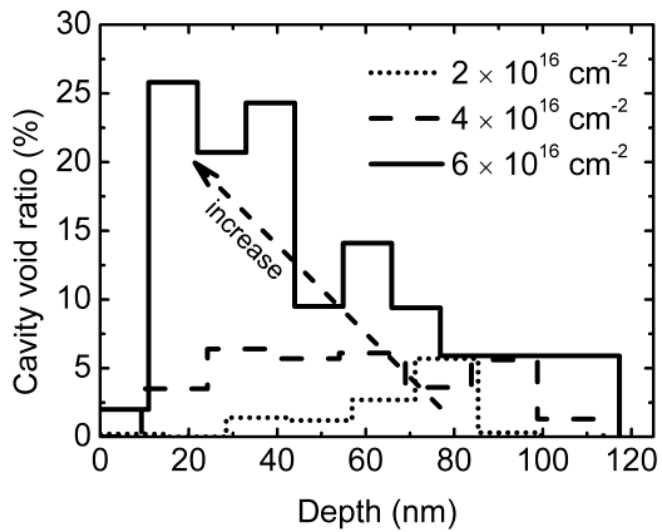

Figure 4: Fluence dependence of the cavity distribution of the implanted $\mathrm{Si} / \mathrm{SiO}^{2}$ samples covered with $130 \mathrm{~nm}$ sacrificial oxide layer and annealed at $800{ }^{\circ} \mathrm{C}$

The last investigated effect on the implanted samples is the influence of the annealing temperature on the evolution of the cavity morphology. Even with three different temperature conditions we can observe a change in the cavity distribution. In Figure 5 the temperature dependence of the cavity distribution of the samples covered with 130 nm thick sacrificial oxide layer and implanted with $4 \times 10^{16} \mathrm{~cm}^{-2}$ dose is shown. There is a sharpening of the cavity volume distribution peak while maintaining the total cavity volume (within $15 \%$ ) when increasing annealing temperature corresponding to the evolution of cavities with temperature following an Ostwald Ripening mechanism [7]. The total cavity volumes are $543,480,571 \mathrm{~nm} \times$ unit surface for the 650,800 and $1000{ }^{\circ} \mathrm{C}$ annealing temperatures respectively. For most of the samples either the appearance of a peak from a rather flat toped cavity void distribution or a sharpening of the existing peak can be observed when increasing temperature.

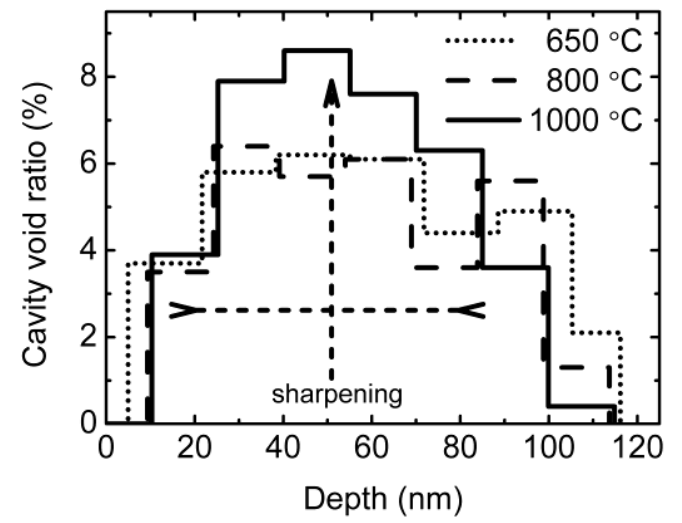

Figure 5: Temperature dependence of the cavity distribution of the $\mathrm{SiO}_{2} / \mathrm{Si}$ samples covered with $130 \mathrm{~nm}$ thick sacrificial oxide layer and implanted with $4 \times 10^{16} \mathrm{~cm}^{-2}$ dose.

\subsection{Effects of implantation and annealing on GaN}

A difficulty of characterizing the GaN thin layers arises because of the shape of its dielectric function. The imaginary part of the complex dielectric function $\left(\varepsilon_{2}\right)$ has a sharp fall at $3.4 \mathrm{eV}(362 \mathrm{~nm})$ for $\mathrm{GaN}$, and so the absorption changes from 0.4 to almost no absorption (for thin layer scales) within a wavelength range of only $20 \mathrm{~nm}$. This causes the optical penetration depth (OPD) to vary from $\sim 50 \mathrm{~nm}$ to several microns in a small spectral width. The drawback is that, at wavelengths where the OPD is larger than the thickness of the first layer from the top of the deposited structure, the non-implanted GaN cannot be used as bulk reference for the EMA modeling of the annealed samples. Furthermore the layers are very thick for ellipsometric evaluations. Because of these reasons, the evaluations have been made at wavelengths ranging up to $370 \mathrm{~nm}$, were it is still reasonable to believe that the structure of the non-implanted $\mathrm{GaN}$ remains unseen by the probing light, and so $\varepsilon_{2}$ obtained from the wavelength-by-wavelength inversion of the ellipsometric angles (pseudo $\varepsilon_{2}-\left\langle\varepsilon_{2}\right\rangle$ ), can be considered as a bulk reference (see Figure 6). This assumption is also confirmed by the fact that the $\left\langle\varepsilon_{2}\right\rangle$ spectra separates for different angles of incidence above $370 \mathrm{~nm}$. In Figure 6 it is also shown that the $\left\langle\varepsilon_{2}\right\rangle$ spectra of $\mathrm{GaN}$ on Sapphire substrate and of GaN on Si substrate are almost identical below $370 \mathrm{~nm}$, but different and straggly above that wavelength. Because of the restriction of useful wavelength range for the evaluations and because of the absorption at these wavelengths, the number of EMA layers fitted is lower than for the implanted Si type samples (model shown in Figure 8 as inset).

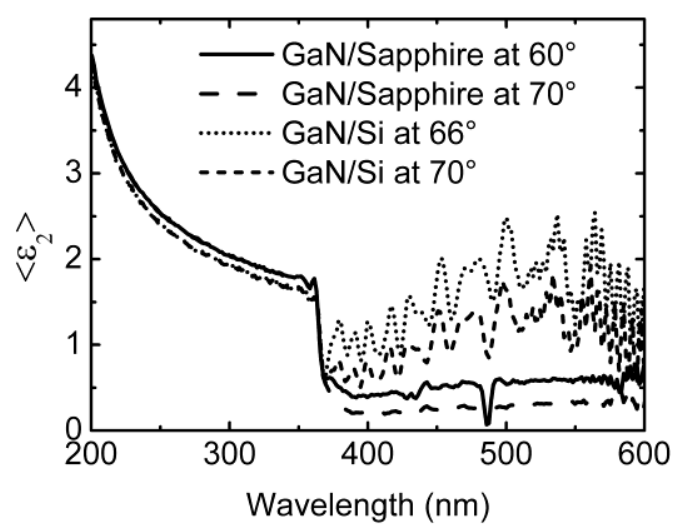

Figure 6: Measured pseudo $\varepsilon_{2}$ of non-implanted GaN/Sapphire and GaN/Si type samples for 2-2 angles of incidence.

In Figure $7\left\langle\varepsilon_{2}\right\rangle$ values are shown for the non-implanted, the asimplanted and for two of the annealed samples of the GaN/Sapphire type. Due to the implantation induced defects, the dielectric function of the GaN changes. The edge of the $\left\langle\varepsilon_{2}\right\rangle$ at $362 \mathrm{~nm}$ disappears and gives rise to a broad peak at $330 \mathrm{~nm}$. After annealing, the shape of the $\left\langle\varepsilon_{2}\right\rangle$ (and of the $\Delta$ ) spectrum starts to look like the one of the non-implanted $\mathrm{GaN}$, evidencing that there is a decrease of the damaged component of the GaN. This decrease of damaged component by increasing temperature is revealed by the fitting of a model of three EMA sublayers. In Figure 8 the damaged GaN component values are shown for the top EMA and middle EMA sublayers as a function of the annealing temperature. Because of the restricted wavelength range, light could not probe the bottom interface of the damaged GaN layer, and the 
evaluations were insensitive to the damaged $\mathrm{GaN}$ component of the bottom sublayer. The TEM images reveal that there is no cavity formation at $[600-900]{ }^{\circ} \mathrm{C}$ temperatures (see Figure 9.a for $900{ }^{\circ} \mathrm{C}$ ) but triangular or elongated shaped cavities appear at $1000{ }^{\circ} \mathrm{C}$ (Figure 9.b). From the SE evaluations of our previous model, it was impossible to estimate a good cavity volume ratio by the addition of void component in the EMA sublayers, so further development of the mode is required, probably with additional reference measurements of nonimplanted and implanted $\mathrm{GaN}$, considering also the use of reference nanocrystalline $\mathrm{GaN}$ in the models similarly as it has been done to $\mathrm{Si}$ based nanostructures $[13,14]$

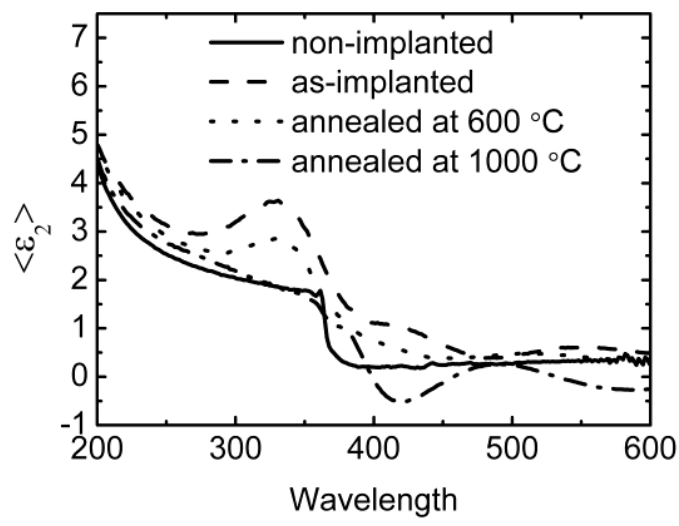

Figure 7: Measured pseudo $\varepsilon_{2}$ of GaN/Sapphire type samples for different treatments.

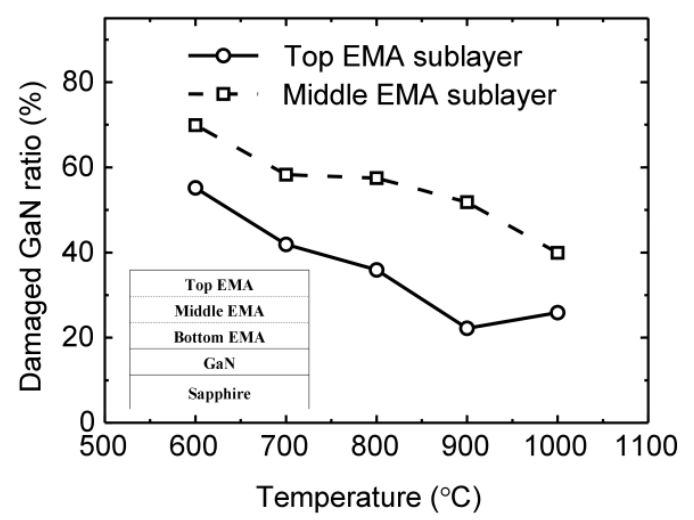

Figure 8: Damaged GaN volume ratio in function of annealing temperature with inset of ellipsometric model used.
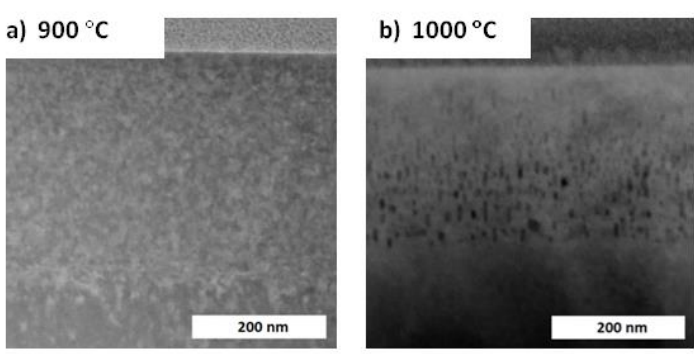

Figure 9: STEM micrographs of implanted and annealed GaN/Sapphire at a) 900 ${ }^{\circ} \mathrm{C}$ and b) $1000{ }^{\circ} \mathrm{C}$.

\section{Conclusions}

In this work, it has been shown that with multilayered effective medium models, the in-depth profile of implantation induced defects, and of annealing induced cavities can be evaluated with a spectroscopic ellipsometer with as good or better depth resolution than with the time consuming analysis of the TEM micrographs. Various implanted Si substrates have been investigated as a function of the implantation dose, the annealing temperature, and the thickness of the sacrificial oxide layer covering the $\mathrm{Si}$. The cavity layer decreases and shifts to the surface with increasing oxide thickness. The cavity volume greatly increases when increasing ion fluence, while the peak of the cavity densities becomes more localized to the surface region. The annealing temperature induces a sharpening of the peak of the cavity distributions while maintaining the total cavity volume nearly unchanged.

Concerning the samples with a thick GaN layer, the investigations are limited by the thickness of the layers and the shape of the dielectric function of the non-implanted GaN. The wavelength-by-wavelength inversion of the ellipsometric angles to obtain a pseudo $\varepsilon_{2}$ could be only used for bulk reference for GaN up to a wavelength of $370 \mathrm{~nm}$. Even with these restriction a simple three sublayered structure revealed that the damage caused by the implantation decreases when increasing annealing temperature, and that there is no cavity formation at annealing temperatures of $900{ }^{\circ} \mathrm{C}$ and below, confirmed with TEM images.

\section{Acknowledgments}

This work was supported by the National Development Agency grants SROP-4.2.2/B-10/1-2010-0029, SROP-4.2.2.B-10/1-2010-0025, OTKA grant Nr. K81842, the Hungarian-French Intergovernmental S\&T Cooperation Programme grant Nr. TÉT_10-1-2011-0754 and by CampusFrance in the frame of a Balaton PHC project.

\section{References}

[1] O. Marcelot, A. Claverie, D. Alquier, F. Cayrel, W. Lerch, S. Paul, L. Rubin, Solid State Phenom. 131-133 (2008) 357.

[2] O. Marcelot, A. Claverie, M. Gavelle, F. Cristiano, F. Cayrel, D. Alquier, W. Lerch, S. Paul, L. Rubin, H. Jaouen, C. Armand, Nucl. Instrum. Methods Phys. Res. B 257 (2007) 249.

[3] F. Cayrel, D. Alquier, C. Dubois and R. Jérisian, Mater. Sci. Eng. B 124 (2005) 271.

[4] F. Cayrel, D. Alquier, D. Mathiot, L. Ventura, F. Roqueta, G. Gaudin and R. Jérisian, Nucl. Instrum. Methods Phys. Res. B 216 (2004) 291.

[5] D. Alquier, F. Roqueta, L. Ventura, F. Cayrel, C. Dubois and R. Jérisian, Jpn. J. Appl. Phys. 41(2002) 3625.

[6] D. Alquier, F. Cayrel, O. Menard, A.-E. Bazin, A. Yvon, E. Collard, Jpn. J. Appl. Phys. 51 (2012) 01AG08.

[7] P. Petrik, M. Fried, T. Lohner, O. Polgár, J. Gyulai, F. Cayrel, and D. Alquier, J. Appl. Phys. 97 (2005) 123514.

[8] P.Petrik, F. Cayrel, M. Fried, O. Polgar, T. Lohner, L. Vincent, D. Alquier, J. Gyulai, Thin Solid Films 455 (2004) 344.

[9] M. Fried, P. Petrik, T. Lohner, N. Q. Khanh, O. Polgar, J. Gyulai, Thin Solid Films 455 (2004) 404

[10] D. Shamiryan, D. V. Likhachev, Mark Goorsky (Ed.), Ion Implantation, Chap. 5, InTech, 2012.

[11] http://www.srim.org/, James F. Ziegler.

[12] T. Lohner, E. Kotai, N.Q. Khanh, Z. Toth, M. Fried, K. Vedam, N.V. Nguyen, L.J. Hanekamp, A. van Silfhout, Nucl. Instrum. Methods Phys. Res. B 85 (1994) 335.

[13] E. Agocs, P. Petrik, M. Fried and A. G. Nassiopoulou, Mater. Res. Soc. Symp. Proc. 1321 (2012) 367.

[14] G. E. Jellison Jr., M. F. Chrisholm, S. M. Gorgatkin, Appl. Phys. Lett. 62 (1993) 3348 